\title{
Power Inverted Nadarajah-Haghighi Distribution: Properties, Estimation, and Applications
}

\author{
Muhammad Ahsan-ul-Haq $\mathbb{D}^{1},{ }^{1}$ Junaid Ahmed $\left(\mathbb{D},{ }^{2}\right.$ Mohammed Albassam $\mathbb{D}^{3},{ }^{3}$ \\ and Muhammad Aslam (iD ${ }^{3}$ \\ ${ }^{1}$ College of Statistical \& Actuarial Sciences, University of the Punjab, Lahore, Pakistan \\ ${ }^{2}$ School of Statistics, Minhaj University Lahore, Lahore, Pakistan \\ ${ }^{3}$ Department of Statistics, Faculty of Science, King Abdulaziz University, Jeddah 21551, Saudi Arabia
}

Correspondence should be addressed to Muhammad Ahsan-ul-Haq; ahsanshani36@gmail.com

Received 17 November 2021; Accepted 20 January 2022; Published 2 March 2022

Academic Editor: Clemente Cesarano

Copyright (C) 2022 Muhammad Ahsan-ul-Haq et al. This is an open access article distributed under the Creative Commons Attribution License, which permits unrestricted use, distribution, and reproduction in any medium, provided the original work is properly cited.

\begin{abstract}
In this paper, a new three-parameter power inverted Nadarajah-Haghighi distribution is proposed and studied. The new distribution is a generalization of the inverted Nadarajah-Haghighi distribution in which an additional shape parameter is included. Surprisingly, this additional shape parameter dominates the tails' weights of distribution and also affects skewness and kurtosis. Such heavy-tailed distributions are suitable for fitting actuarial and financial risk real problems. Important mathematical properties of this distribution are obtained. Characterization based on two truncated moments is also derived. The parameters are estimated using the maximum likelihood method. Further, a numerical simulation study is conducted for reinforcement of the results. Finally, the application of the proposed distribution to real-life data sets is presented to prove its applicability.
\end{abstract}

\section{Introduction}

The theory of probability distribution has been contributing a lot to deal with the small as well as large datasets. The models of the probability distribution are of much importance and used extensively in various fields like healthcare, engineering, and business management. Additionally, the study of social sciences like economics and psychology are also covered in it. They are best suited for prediction and forecast in the modelling of real-world problems.

An extension of the exponential distribution known as Nadarajah-Haghighi distribution was developed by Nadarajah and Haghighi [1]. It is a generalized form of exponential distribution and is used in place of Weibull, exponentiated exponential, and gamma distributions. Its unique feature is that it always has modes of zero. Hence, it allows for variable hazard rate functions, i.e., increasing, decreasing, or constant.

For the past decades, various traditional distributions are being used broadly for the demonstration of datasets in many fields. Sometimes, these distributions do not provide a good fit for heavy-tailed and more skewed datasets. So, it was essentially needed to produce extended forms of these models for the handling of such datasets. Consequently, in order to derive new probability distributions, various methods have been proposed.

Tahir, Cordeiro, Ali, Dey, and Manzoor [2] proposed the inverted Nadarajah-Haghighi (INH) distribution. AbdulMoniem [3] introduced an extended form of the Nadarajah and Haghighi exponential distribution called Exponentiated Nadarajah and Haghighi Exponential (ENHE) distribution. The ENHE distribution was applied to real data and showed that the new distribution gave effective results as compared with the Nadarajah-Haghighi distribution. VedoVatto and others [4] developed an Exponentiated Generalized Nadarajah-Haghighi distribution. The proposed distribution is flexible due to its hazard rate behavior which can be constant, decreasing, increasing, unimodal, bathtub-shaped, and upside-down bathtub forms. Korkmaz and Yousof [5] developed Topp-Leone Nadarajah-Haghighi distribution. 
This distribution can be used in the modelling of survival data, reliability problems, and fatigue life studies. Ogunde, Ibraheem, and Audu [6] proposed transmuted Nadarajah and Haghighi distribution. Dias, Alizadeh, and Cordeiro [7] developed an extended model called the Beta Nadarajah-Haghighi (BNH) distribution. Toumaj and Mostafaee [8] introduced transmuted inverted Nadarajah-Haghighi distribution. Rafiq and others [9] proposed Marshall-Olkin inverted Nadarajah-Haghighi distribution.

In this study, we develop an extension of the INH distribution called power inverted Nadarajah-Haghighi distribution. The study is organized as follows. We derive the proposed distribution and its limiting behavior in Section 2. Mathematical properties such as moments, probability weighted moments, moment generating function, incomplete moments, and order statistics are derived in Section 3. In Section 4, different entropies are obtained. Reliability measures, mean residual life, reversed residual life, and strong mean Inactivity time are given in Section 5. Characterization based on truncated moments in Section 6. The distributional parameters are estimated by the method of maximum likelihood in Section 7. Section 8 is devoted to a detailed simulation study. A real-life application is presented in Section 9, and the concluding remarks are given in Section 10.

\section{Power Inverted Nadarajah-Haghighi (PINH) Distribution}

A new extension of the INH distribution is introduced using power transformation $X=Z^{1 / \gamma}$, where $Z$ is random which has two parameters $(\alpha, \beta)$. Let $X$ be a nonnegative random variable that follows PINH distribution, i.e., $X \sim \operatorname{PINH}(\alpha, \beta, \gamma)$, then cumulative distribution function (cdf) and the probability density function (pdf) are given.

$$
\begin{gathered}
F(x)=e^{\left\{1-\left(1+\beta / x^{\gamma}\right)^{\alpha}\right\}} \gamma, \alpha, \beta,>0, x>0, \\
f(x)=\alpha \beta \gamma x^{-\gamma-1}\left(1+\frac{\beta}{x^{\gamma}}\right)^{\alpha-1} e^{\left\{1-\left(1+\beta / x^{\gamma}\right)^{\alpha}\right\}},
\end{gathered}
$$

where $\beta$ represents the scaling factor and $(\alpha, \gamma)$ represents the shape of the curve. Figure 1 presents the density plots of the pdf of PINH distribution for different parameter values.

The alternative form of density function is as follows.

By using the expansion $e^{-x}=\sum_{m=0}^{\infty}(-1)^{m} x^{m} / m$ !, we can write $e^{-\left(1+\beta / x^{\gamma}\right)^{\alpha}}=\sum_{m=0}^{\infty}(-1)^{m} / m !\left(1+\beta / x^{\gamma}\right)^{\alpha m}$.

And,

$$
f(x)=e \alpha \beta \gamma x^{-\gamma-1} \sum_{m=0}^{\infty} \frac{(-1)^{m}}{m !}\left(1+\frac{\beta}{x^{\gamma}}\right)^{\alpha m+\alpha-1} .
$$

\section{Mathematical Properties}

The quantile function of PINH distribution can be obtained by inverting equation (1) and is given by

$$
x=\left[\frac{[1.693]^{1 / \alpha}-1}{\beta}\right]^{-1 / \gamma} .
$$

If the random variable $X$ is PINH distributed, then its $\mathrm{r}^{\text {th }}$ moment around zero can be expressed as

$$
\mu_{r}^{\prime}=e \alpha \sum_{m=0}^{\infty} \frac{(-1)^{m}}{m !} \beta^{r / \gamma} B\left[\left(1-\frac{r}{\gamma}\right),\left(\frac{r}{\gamma}-\alpha(m+1)\right)\right],
$$

where $B(\cdot, \cdot)$ is the beta function.

Another important function is the moment generating function of PINH distribution which can be obtained as

$$
\begin{aligned}
M o(t)= & e \alpha \beta^{i / \gamma} \cdot \sum_{i=0}^{\infty} \frac{(t)^{i}}{i !} \sum_{r=0}^{\infty} \frac{(-1)^{r}}{r !} \\
& \cdot B\left[\left(1-\frac{i}{\gamma}\right),\left(\frac{i}{\gamma}-\alpha(r+1)\right)\right] i<\gamma .
\end{aligned}
$$

The incomplete moment of PINH distribution can be obtained as follows:

$\phi_{r}=e \alpha \beta^{r / \gamma} \sum_{i=0}^{\infty} \frac{(-1)^{i}}{i !} B\left[\left(1-\frac{r}{\gamma}\right),\left(\frac{r}{\gamma}-\alpha(i+1)\right)\right], \quad r<\gamma$.

The $\mathrm{r}^{\text {th }}$ probability weighted moment of the residual life of the PINH distribution is

$$
\begin{aligned}
B_{K}= & \alpha \beta^{1 / \gamma} e^{k+1} k^{j} \sum_{i=0}^{\infty} \frac{(-1)^{i}}{i !} \sum_{j=0}^{\infty} \frac{(-1)^{j}}{j !} \\
& \cdot \beta\left[1-\frac{1}{\gamma}, \frac{1}{\gamma}-\alpha(1+i+j)\right] .
\end{aligned}
$$

It is actually the scattering of sample values and ultimately shows the scattering in the population. The sample values are placed in ascending order. They are denoted by $X_{(1)}, X_{(2) \ldots} X_{(\mathrm{n})}$.

The minimum order statistic of PINH distribution is given by

$$
\begin{aligned}
& f_{1}(x)=n \alpha \beta \gamma x^{-\gamma-1}\left(1+\frac{\beta}{x^{\gamma}}\right)^{\alpha-1} \\
& \cdot e^{\left[1-\left(1+\beta / x^{\gamma}\right)^{\alpha}\right]}\left[1-e^{\left[1-\left(1+\beta / x^{\gamma}\right)^{\alpha}\right]}\right]^{n-1} \cdot
\end{aligned}
$$

The maximum order statistic of PINH distribution is obtained as

$$
f_{n}(x)=n \alpha \beta \gamma x^{-\gamma-1}\left(1+\frac{\beta}{x^{\gamma}}\right)^{\alpha-1}\left[e^{n\left[1-\left(1+\beta / x^{\gamma}\right)^{\alpha}\right]}\right] .
$$

\section{Entropies}

4.1. Rényi Entropy. Rényi entropy is simply an index of diversity. Rényi entropy is developed by Hungarian mathematician [10]. 

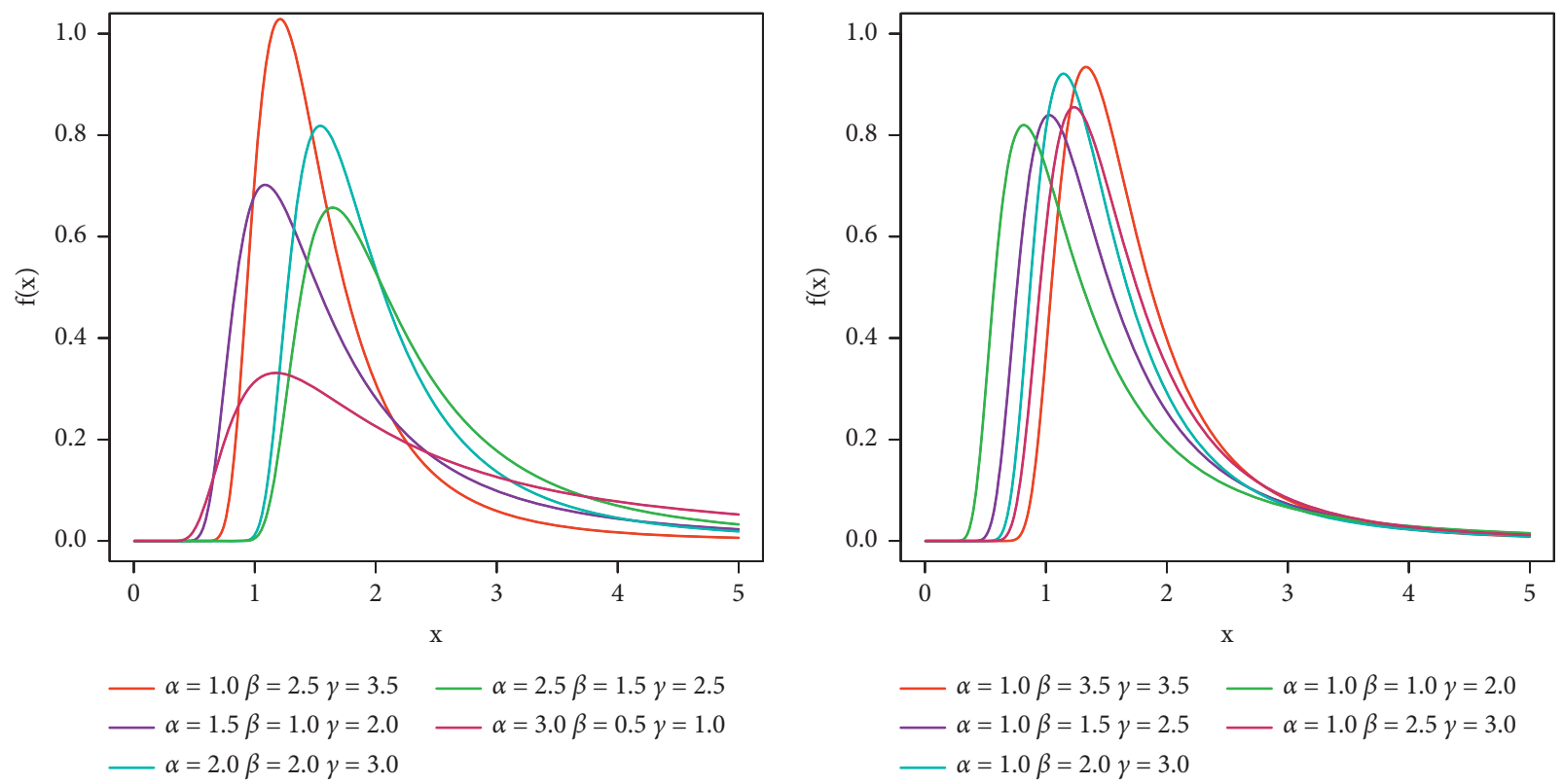

FIgURE 1: Pdf curves of PINH distribution for some parameter values.

$$
H_{R}(S)=\frac{1}{1-\gamma} \ln \left[\frac{e^{s}(\alpha \gamma)^{s} \beta^{-s / \gamma+1 / \gamma}}{\gamma} \sum_{i=0}^{\infty} \frac{(-1)^{i} s^{i}}{i !} B\left[\left(s+\frac{s}{\gamma}-\frac{1}{\gamma}\right),\left(\frac{1}{\gamma}-\frac{s}{\gamma}-s \alpha-a i\right)\right]\right] .
$$

\subsection{Tsallis Entropy}

$$
S_{q}(x)=\frac{1}{q-1} \ln \left[1-\frac{e^{q}(\alpha \gamma)^{q} \beta^{-q / \gamma+1 / \gamma}}{\gamma} \sum_{i=0}^{\infty} \frac{(-1)^{i} q^{i}}{i !} B\left[\left(q+\frac{q}{\gamma}-\frac{1}{\gamma}\right),\left(\frac{1}{\gamma}-\frac{q}{\gamma}-q \alpha-a i\right)\right] .\right.
$$

\section{Reliability Analysis}

$$
r(t)=\left[\alpha \beta \gamma x^{-\gamma-1}\left(1+\frac{\beta}{x^{\gamma}}\right)^{\alpha-1}\right]
$$

The survival and hazard rate functions of PINH distribution are given by

$$
\begin{aligned}
& R(x)=1-e^{\left[1-\left(1+\beta / x^{\gamma}\right)^{\alpha}\right]}, \quad \alpha, \gamma, \beta>0, x>0, \\
& h(x)=\frac{\alpha \beta \gamma x^{-\gamma-1}\left(1+\beta / x^{\gamma}\right)^{\alpha-1} e^{\left[1-\left(1+\beta / x^{\gamma}\right)^{\alpha}\right]}}{1-e^{\left[1-\left(1+\beta / x^{\gamma}\right)^{\alpha}\right]}} .
\end{aligned}
$$

Figure 2 presents the shapes of the hazard function of PINH distribution for different parameter values.

The expression of the reversed hazard rate function is

5.1. Mean of Residual Life and Reversed Residual Life. Given that a segment survives up to time $t \geq 0$, the residual life is the period beyond $t$ until the time of failure and characterized by the expectation of the conditional random variable $(X \mid X>t)$. In reliability, it is well known that the mean residual life function and the ratio of two consecutive moments of residual life determine the distribution uniquely. Therefore, we obtain the mean of the residual life of $X$ : 

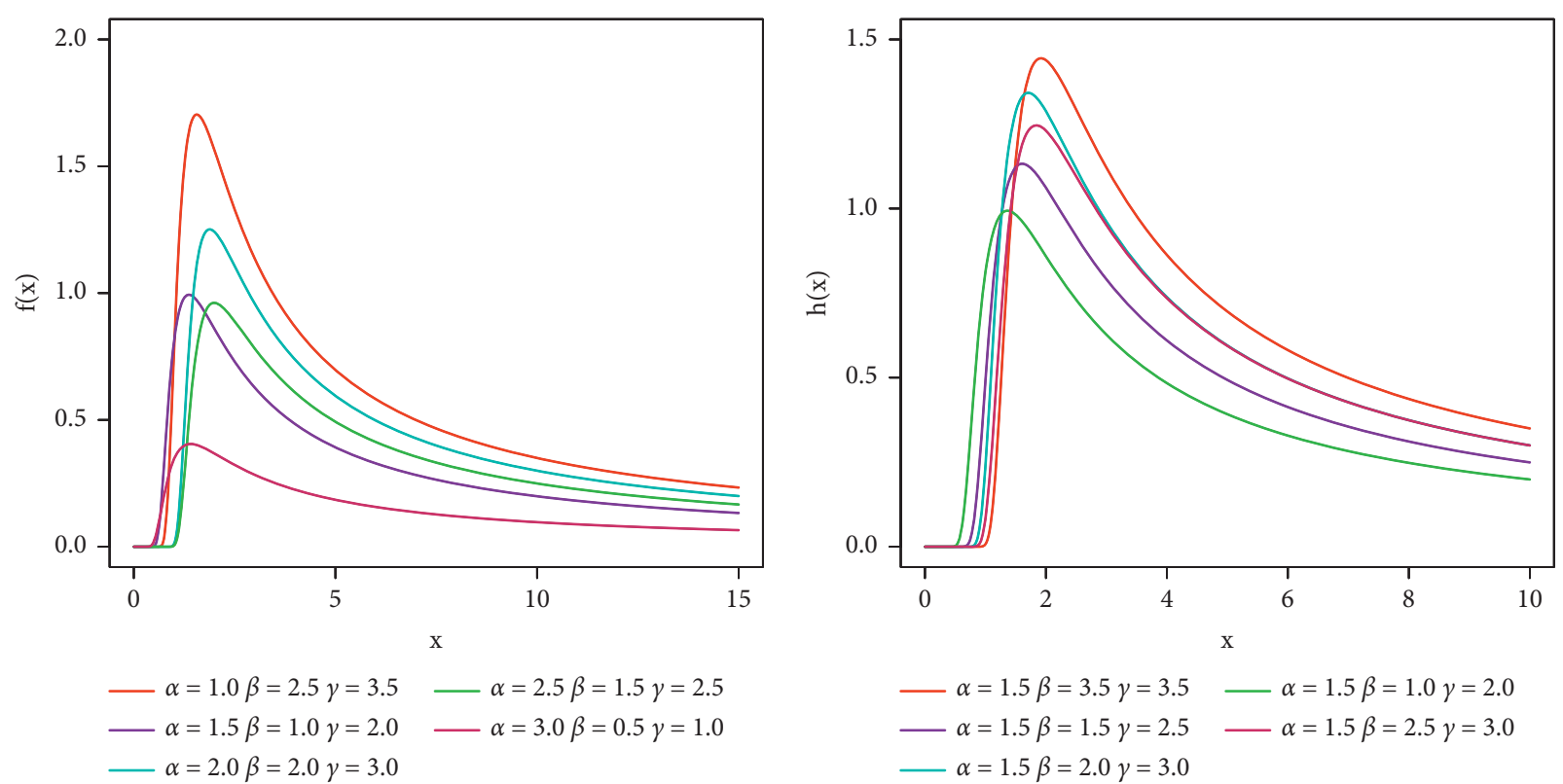

FIGURE 2: Hrf curves of PINH distribution for some parameter values.

$$
\begin{aligned}
& \mu(t)=E((X-t) \mid X>t)=\frac{1}{1-F(t)} \int_{t}^{\infty}(x-t) f(x) \mathrm{d} x, \\
& \mu(t)=\frac{e \alpha \beta^{1 / \gamma}}{1-e^{\left[1-\left(1+\beta / t^{\gamma}\right)^{\alpha}\right]}} \sum_{r=0}^{\infty} \frac{(-1)^{r}}{r !} \beta\left[1-\frac{1}{\gamma}, \frac{1}{\gamma}-\alpha-\alpha r\right]-t .
\end{aligned}
$$

The mean inactivity time (MIT) function is the mean of idle time that has passed away. It is used for the analysis of survival functions and is also used in reliability theory. The MIT function is defined as

$$
m(t)=t-\frac{e \alpha \beta^{1 / \gamma}}{e^{\left[1-\left(1+\beta / t^{\gamma}\right)^{\alpha}\right]}} \sum_{k=0}^{\infty} \frac{(-1)^{k}}{k !} \beta\left[1-\frac{1}{\gamma}, \frac{1}{\gamma}-\alpha-\alpha k\right] .
$$

5.2. The Strong Mean Inactivity Time. The strong mean inactivity time is proposed by [11]. The strong mean inactivity time of a random variable $X$ is defined as

$$
\begin{aligned}
& M(t)=t^{2}-\frac{1}{G(t)} \int_{0}^{t} x^{2} g(x) \mathrm{d} x, \quad t>0, \\
& M(t)=t^{2}-\frac{e \alpha \beta^{2 / \gamma}}{e^{\left[1-\left(1+\beta / t^{\gamma}\right)^{\alpha}\right]}} \sum_{k=0}^{\infty} \frac{(-1)^{k}}{k !} \beta\left[1-\frac{2}{\gamma}, \frac{2}{\gamma}-\alpha-a k\right] 2<\gamma .
\end{aligned}
$$

\section{Characterizations}

6.1. Characterizations Based on Two Truncated Moments. In this subsection, characterizations of PINH distribution based on the ratio of two truncated moments are presented. For characterization of distribution, we employ Glanzel approach [12] presented in Appendix A.

Proposition 1. Let $X: \Omega \longrightarrow(0, \infty)$ be distributed as equation (2), and let

$$
\begin{aligned}
& q_{1}(x)=\operatorname{Exp}\left[-\left\{1-\left(1+\frac{\beta}{x^{\gamma}}\right)^{\alpha}\right\}\right] \\
& q_{2}(x)=q_{1}(x)\left(1+\frac{\beta}{x^{\gamma}}\right)^{\alpha}, \quad x>0 .
\end{aligned}
$$

The continuous random variable $X$ follows PINH distribution if and only if the function $\eta$ defined in Theorem 1 (Appendix A) has of the form as follows:

$$
\eta(x)=\frac{1}{2}\left(1+\frac{\beta}{x^{\gamma}}\right)^{\alpha}, \quad x>0 .
$$


Proof. If $X$ has pdf (2), then

$$
\begin{aligned}
& (1-F(x)) E\left[q_{1}(X) \mid X \geq x\right]=\int_{x}^{\infty} \alpha \beta \gamma x^{-\gamma-1}\left(1+\frac{\beta}{x^{\gamma}}\right)^{\alpha-1} e^{\left[1-\left(1+\beta / x^{\gamma}\right)^{\alpha}\right]} * \operatorname{Exp}\left[-\left\{1-\left(1+\frac{\beta}{x^{\gamma}}\right)^{\alpha}\right\}\right] \mathrm{d} x, \\
& (1-F(x)) E\left[q_{1}(X) \mid X \geq x\right]=\left(1+\frac{\beta}{x^{\gamma}}\right)^{\alpha}-1, \quad x>0 .
\end{aligned}
$$

Similarly,

$(1-F(x)) E\left[q_{2}(X) \mid X \geq x\right]=\frac{1}{2}\left(\left(1+\frac{\beta}{x^{\gamma}}\right)^{2 \alpha}-1\right), \quad x>0$.

$$
\eta(x)=\frac{E\left[q_{2}(X) \mid X \geq x\right]}{E\left[q_{1}(X) \mid X \geq x\right]}=\frac{1}{2}\left\{\left(1+\frac{\beta}{x^{\gamma}}\right)^{\alpha}+1\right\} .
$$

This completes the proof.

Conversely, if $\eta$ is given as above (19), then

Now,

$$
\begin{aligned}
\eta(x) q_{1}(x)-q_{2}(x) & =q_{1}(x)\left[\eta(x)-\operatorname{Exp}\left[-\lambda\left(\frac{x^{\theta}}{1-x^{\beta}}\right)\right]\right] \\
& =-\frac{1}{2} \operatorname{Exp}\left[-\left\{1-\left(1+\frac{\beta}{x^{\gamma}}\right)^{\alpha}\right\}\right]\left(\left(1+\frac{\beta}{x^{\gamma}}\right)^{\alpha}-1\right)<0 \\
s^{\prime}(x) & =\frac{\eta^{\prime}(x) q_{1}(x)}{\eta(x) q_{1}(x)-q_{2}(x)}=\frac{\mathrm{e}^{-1+\left(1+\beta / x^{\gamma}\right)^{\alpha}} x^{-(1+\gamma)} \alpha \beta\left(1+\beta / x^{\gamma}\right)^{\alpha-1} \gamma}{2\left[-\mathrm{e}^{-1+\left(1+\beta / x^{\gamma}\right)^{\alpha}}\left(1+\beta / x^{\gamma}\right)^{\alpha}+1 / 2 \mathrm{e}^{\left(1+\beta / x^{\gamma}\right)^{\alpha}}\left\{1+\left(1+\beta / x^{\gamma}\right)^{\alpha}\right\}\right]} \\
& =\frac{\alpha \beta\left(1+\beta / x^{\gamma}\right)^{\alpha} \gamma}{x\left(x^{\gamma}+\beta\right)\left[\left(1+\beta / x^{\gamma}\right)^{\alpha}-1\right]},
\end{aligned}
$$

and hence

$$
s(x)=-\ln \left(\left(1+\frac{\beta}{x^{\gamma}}\right)^{\alpha}-1\right), \quad x>0 .
$$

Now, by Theorem 1 (Appendix A), $X$ has density equation (2).

Corollary 2. Let $X: \Omega \longrightarrow(0, \infty)$ be a continuous random variable, and let $q_{1}(x)$ be as in Proposition 1. The pdf of $X$ is
(2) if and only if there exist functions $q_{2}(x)$ and $\eta$ defined in Theorem 1 (Appendix A) satisfying the differential equation:

$$
\frac{\eta^{\prime}(x) q_{1}(x)}{\eta(x) q_{1}(x)-q_{2}(x)}=\frac{\alpha \beta\left(1+\beta / x^{\gamma}\right)^{\alpha} \gamma}{x\left(x^{\gamma}+\beta\right)\left[\left(1+\beta / x^{\gamma}\right)^{\alpha}-1\right]}, \quad x>0 .
$$

The general solution of the differential equation (25) is

$$
\eta(x)=\left[\left(1+\frac{\beta}{x^{\gamma}}\right)^{\alpha}-1\right]^{-1}\left[\alpha \beta \gamma \int_{0}^{x}\left(1+\frac{\beta}{x^{\gamma}}\right)^{\alpha}\left(x\left(x^{\gamma}+\beta\right)\right)^{-1}\left[q_{2}(x)\left\{q_{1}(x)\right\}^{-1}\right] \mathrm{d} x+D\right],
$$

where $D$ is a constant. Note that a set of functions satisfying the differential equation (25) is given in Theorem 1 with $D=0$. However, it should also be noted that there are other triplets $\left(q_{1}, q_{2}, \eta\right)$ satisfying the condition of Theorem 1.

\section{Maximum Likelihood Estimation}

Let $x_{1}, x_{2}, \ldots, x_{n}$ be random samples distributed according to the PINH distribution, the likelihood function is obtained by the relationship as follows: 
TABLE 1: Estimates of parameter, bias, and MSE at $\alpha=1.0, \beta=1.5$, and $\gamma=1.5$.

\begin{tabular}{lcccc}
\hline Sample size & Parameters & Estimates & Bias & MSE \\
\hline \multirow{3}{*}{80} & $\alpha$ & 4.2093 & 3.2093 & 226.33 \\
& $\beta$ & 2.4130 & 0.9130 & 70.591 \\
& $\gamma$ & 1.5555 & 0.0555 & 0.1117 \\
\hline \multirow{3}{*}{100} & $\alpha$ & 3.2234 & 2.2234 & 138.06 \\
& $\beta$ & 2.1835 & 0.6835 & 22.965 \\
& $\gamma$ & 1.5430 & 0.0430 & 0.0869 \\
\multirow{3}{*}{150} & $\alpha$ & 1.6996 & 0.6996 & 21.559 \\
& $\beta$ & 1.8030 & 0.3030 & 3.0009 \\
\multirow{3}{*}{200} & $\gamma$ & 1.5232 & 0.0232 & 0.0511 \\
\hline \multirow{3}{*}{300} & $\alpha$ & 1.3400 & 0.3400 & 4.0648 \\
& $\beta$ & 1.7253 & 0.2253 & 1.4737 \\
& $\gamma$ & 1.5206 & 0.0206 & 0.0390 \\
\hline
\end{tabular}

$$
L=\prod_{i=1}^{n} f\left(x_{i}, \alpha, \beta, \gamma\right)
$$

Using the expression in equation (2), we have

$$
L=\prod_{i=1}^{n} \alpha \beta \gamma x^{-\gamma-1}\left(1+\frac{\beta}{x^{\gamma}}\right)^{\alpha-1}\left[e^{\left[1-\left(1+\beta / x^{\gamma}\right)^{\alpha}\right]}\right] .
$$

By taking the natural logarithm, the log-likelihood function is obtained as
TABLE 2: Estimates of parameter, bias, and MSE at $\alpha=0.5, \beta=0.5$, and $\gamma=0.5$.

\begin{tabular}{lcccc}
\hline Sample size & Parameters & Estimates & Bias & MSE \\
\hline \multirow{3}{*}{80} & $\alpha$ & 0.7888 & 0.2888 & 15.082 \\
& $\beta$ & 0.7029 & 0.2029 & 61.125 \\
& $\gamma$ & 0.5275 & 0.0275 & 0.0213 \\
\hline \multirow{3}{*}{100} & $\alpha$ & 0.6148 & 0.1148 & 1.9645 \\
& $\beta$ & 0.5438 & 0.0438 & 0.1231 \\
& $\gamma$ & 0.5207 & 0.0207 & 0.0130 \\
150 & $\alpha$ & 0.5477 & 0.0477 & 0.0484 \\
& $\beta$ & 0.5207 & 0.0207 & 0.0521 \\
\multirow{3}{*}{200} & $\gamma$ & 0.5119 & 0.0119 & 0.0073 \\
\hline \multirow{3}{*}{300} & $\alpha$ & 0.7888 & 0.2888 & 15.082 \\
& $\beta$ & 0.7029 & 0.2029 & 61.125 \\
& $\gamma$ & 0.5275 & 0.0275 & 0.0213 \\
\hline & $\alpha$ & 0.6148 & 0.1148 & 1.9645 \\
& $\beta$ & 0.5438 & 0.0438 & 0.1231 \\
& $\gamma$ & 0.5207 & 0.0207 & 0.0130 \\
\hline
\end{tabular}

$$
\begin{aligned}
\ln (L)= & \alpha \beta \gamma(-\gamma-1) \sum_{i=1}^{n} \ln x+(\alpha-1) \\
& \cdot \sum_{i=1}^{n} \ln \left(1+\frac{\beta}{x^{\gamma}}\right)+\sum_{i=1}^{n}\left[1-\left(1+\frac{\beta}{x^{\gamma}}\right)^{\alpha}\right] .
\end{aligned}
$$

The maximum likelihood estimators $\widehat{\alpha}, \widehat{\gamma}$, and $\widehat{\beta}$ of the unknown parameters $\alpha, \gamma$, and $\beta$ are derived from the nonlinear following score equations as follows:

$$
\begin{aligned}
& \frac{\partial L}{\partial \alpha}=\beta \gamma(-\gamma-1) \sum_{i=1}^{n} \ln x+\sum_{i=1}^{n} \ln \left(1+\frac{\beta}{x^{\gamma}}\right)-\left(1+\frac{\beta}{x^{\gamma}}\right)^{\alpha} \sum_{i=1}^{n} \ln \left(1+\frac{\beta}{x^{\gamma}}\right), \\
& \frac{\partial L}{\partial \beta}=\alpha \gamma(-\gamma-1) \sum_{i=1}^{n} \ln x+(\alpha-1) \sum_{i=1}^{n} \ln x^{-\gamma}-\alpha \sum_{i=1}^{n}\left(1+\frac{\beta}{x^{\gamma}}\right)^{\alpha-1} x^{-\gamma}, \\
& \frac{\partial L}{\partial \gamma}=-2 \alpha \beta \gamma \sum_{i=1}^{n} \ln x-\alpha \beta \sum_{i=1}^{n} \ln x-(\alpha-1) \sum_{i=1}^{n} \frac{\beta \ln x}{\left(1+\beta / x^{\gamma}\right) x^{\gamma}}-\alpha \sum_{i=1}^{n}\left(1+\frac{\beta}{x^{\gamma}}\right)^{\alpha-1} \frac{\beta}{x^{\gamma}} \ln x .
\end{aligned}
$$

Although the nonlinear equations do not have closedform expression, we can obtain the estimates of unknown parameters. To do so, $R$-programming language has been used to get unique values of parameters.

\section{Simulation Studies}

For the support of this research, we conduct a comprehensive simulation study to assess the performance of MLE in estimating the parameters of PINH distribution. For this purpose, the data were simulated from PINH distribution by considering the following parametric values: $\alpha=1, \beta=1.5$, and $\gamma=1.5 ; \alpha=0.5, \beta=0.5$, and $\gamma=0.5$; and $a=0.5, \beta=0.1$, and $\gamma=1.5$. The sample sizes used in this simulation study are $n=80,100,150,200$, and 300. This simulation is repeated for 5000 pseudorandom samples found based on 5000 simulation runs. The performance of MLE is evaluated based on mean square error (MSE) and bias. For a given true value of the PINH parameters, say $\theta$, the MSE and bias are given by the following:

$$
\begin{aligned}
\text { MSE } & =\frac{1}{N} \sum_{i=1}^{n}\left(\widehat{\theta}_{i}-\theta\right)^{2}, \\
\text { Bias } & =\frac{1}{N} \sum_{i=1}^{n}\left(\widehat{\theta}_{i}-\theta\right),
\end{aligned}
$$

where $\widehat{\theta}_{i}$ is the estimated parameter of PINH distribution for the $i$ th $(i=1,2, \ldots, N)$ simulated sample and $N$ denotes the number of simulation runs.

From Tables 1-3, it is found that the average bias and MSEs decrease as sample size increases. It expresses that on 
TABLE 3: Estimates of parameter, bias, and MSE at $\alpha=0.5, \beta=0.1$, and $\gamma=1.5$.

\begin{tabular}{|c|c|c|c|c|}
\hline Sample size & Parameters & Estimates & Bias & MSE \\
\hline \multirow{3}{*}{80} & $\alpha$ & 0.8309 & 0.3309 & 20.108 \\
\hline & $\beta$ & 0.0928 & -0.0072 & 0.0017 \\
\hline & $\gamma$ & 1.5815 & 0.0815 & 0.1772 \\
\hline \multirow{3}{*}{100} & $\alpha$ & 0.6681 & 0.1681 & 6.1941 \\
\hline & $\beta$ & 0.0937 & -0.0063 & 0.0013 \\
\hline & $\gamma$ & 1.5547 & 0.0547 & 0.1198 \\
\hline \multirow{3}{*}{150} & $\alpha$ & 0.5560 & 0.0560 & 0.1493 \\
\hline & $\beta$ & 0.0962 & -0.0038 & 0.0008 \\
\hline & $\gamma$ & 1.5302 & 0.0302 & 0.0612 \\
\hline \multirow{3}{*}{200} & $\alpha$ & 0.5345 & 0.0345 & 0.0255 \\
\hline & $\beta$ & 0.0971 & -0.0029 & 0.0006 \\
\hline & $\gamma$ & 1.5215 & 0.0215 & 0.0291 \\
\hline \multirow{3}{*}{300} & $\alpha$ & 0.5192 & 0.0192 & 0.0141 \\
\hline & $\beta$ & 0.0984 & -0.0016 & 0.0004 \\
\hline & $\gamma$ & 1.5164 & 0.0164 & 0.0291 \\
\hline
\end{tabular}

TABle 4: ML estimates and goodness-of-fit measures for the first data set.

\begin{tabular}{|c|c|c|c|c|c|}
\hline Model & MLE & $-\log L$ & AIC & $\mathrm{BIC}$ & $\mathrm{K}-\mathrm{S}$ ( $p$ value $)$ \\
\hline PINH & $\begin{array}{l}\widehat{\alpha}=1.1919 \\
\widehat{\beta}=3231.8 \\
\widehat{\gamma}=2.3392\end{array}$ & 215.098 & 436.196 & 441.810 & $0.0706(0.9700)$ \\
\hline TINH & $\begin{array}{l}\widehat{\alpha}=60.797 \\
\widehat{\beta}=0.5637 \\
\widehat{\gamma}=0.9999\end{array}$ & 215.666 & 437.332 & 442.946 & $0.0753(0.9500)$ \\
\hline MOINH & $\begin{array}{l}\widehat{\alpha}=0.9329 \\
\widehat{\beta}=172.29 \\
\widehat{\gamma}=0.0262\end{array}$ & 216.597 & 439.194 & 444.808 & $0.0762(0.9400)$ \\
\hline INH & $\begin{array}{l}\widehat{\alpha}=126.62 \\
\widehat{\beta}=0.2217\end{array}$ & 224.904 & 453.808 & 457.55 & $0.2440(0.0065)$ \\
\hline
\end{tabular}

TABle 5: ML estimates and goodness-of-fit measures for the first data set.

\begin{tabular}{|c|c|c|c|c|c|}
\hline Model & MLE & $-\log L$ & AIC & $\mathrm{BIC}$ & $\mathrm{K}-\mathrm{S}$ ( $p$ value $)$ \\
\hline PINH & $\begin{array}{l}\widehat{\alpha}=0.1422 \\
\widehat{\beta}=8119.4 \\
\widehat{\gamma}=2.9655\end{array}$ & 414.126 & 834.252 & 842.808 & $0.0777(0.4200)$ \\
\hline TINH & $\begin{array}{c}\widehat{\alpha}=0.5026 \\
\widehat{\beta}=5.3660 \\
\widehat{\gamma}=-0.8624\end{array}$ & 426.538 & 859.080 & 867.636 & $0.1900(0.0530)$ \\
\hline MOINH & $\begin{aligned} \widehat{\alpha} & =0.9329 \\
\widehat{\beta} & =5756.1 \\
\widehat{\gamma} & =0.0074\end{aligned}$ & 423.644 & 853.288 & 861.844 & $0.0974(0.3900)$ \\
\hline INH & $\begin{aligned} \widehat{\alpha} & =0.5064 \\
\widehat{\beta} & =10.598\end{aligned}$ & 431.06 & 866.12 & 871.82 & $0.12400(0.0400)$ \\
\hline
\end{tabular}

increasing sample size, the value of estimated parameters, bias, and MSE is improved and both bias and MSE are decreased. The results are presented in Tables 1-3; we can observe that the mean squared errors are very small, which confirm the convergence of the maximum likelihood estimators.

\section{Applications}

In this section, we fit the PINH distribution to two sets of real-world data from different fields. The PINH distribution is compared with Marshall-Olkin inverted Nadarajah-Haghighi (MOINH), transmuted inverted Nadarajah-Haghighi (TNH), and inverted Nadarajah-Haghighi (INH) distributions.

The first dataset consists of the annual flood discharges of the North Saskatchewan in units of $1000 \mathbf{f}^{3} /$ second, of the River at Edmonton, over 47 years which is reported by Van Montfort [13]. The values of data are $\{19.885,20.940,21.820$, $23.700,24.888,25.460,25.760,26.720,27.500,28.100,28.600$, $30.200,30.380,31.500,32.600,32.680,34.400,35.347,35.700$, 

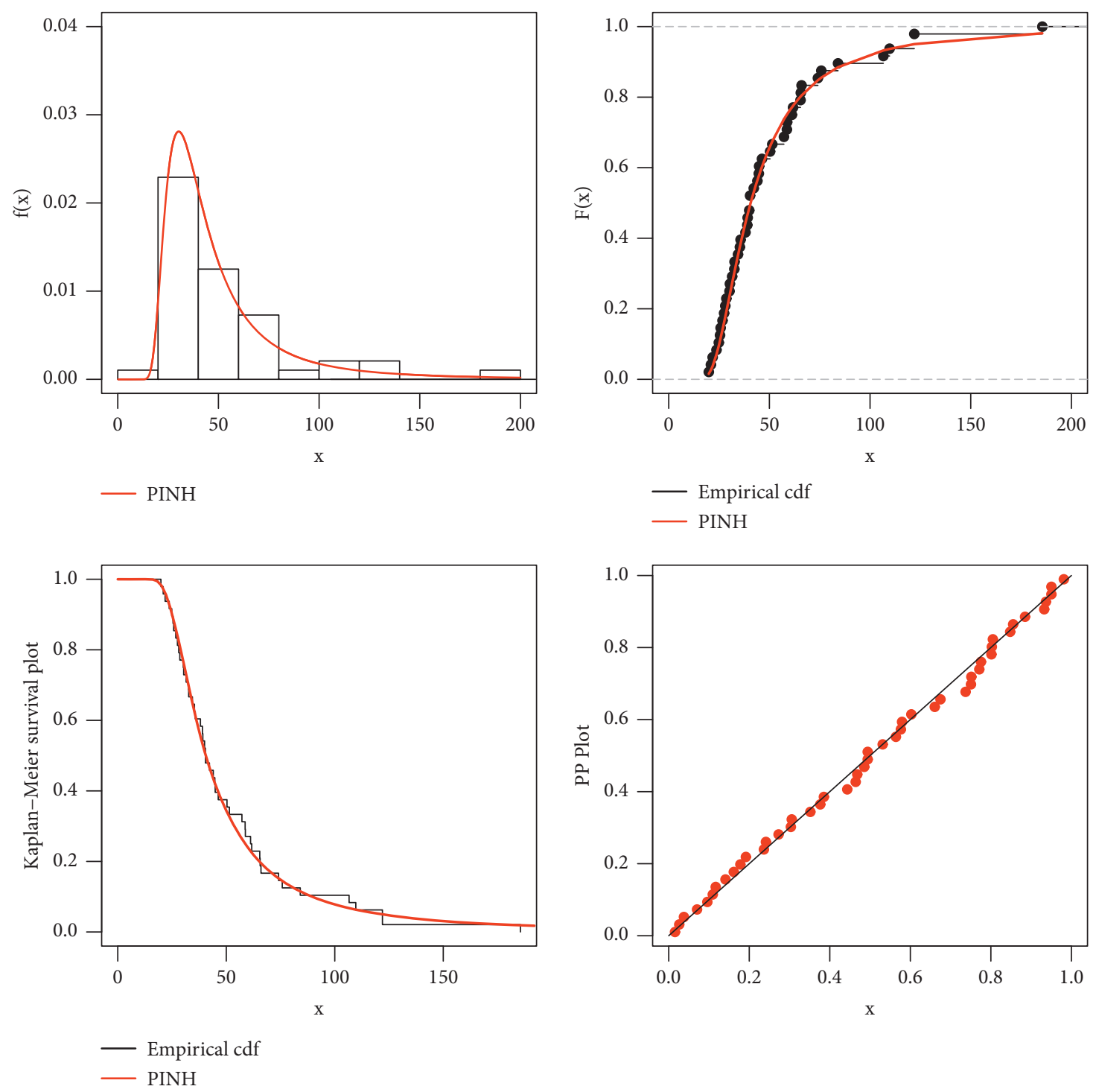

Figure 3: Fitted probability models and empirical data for first data set.

38.100, 39.020, 39.200, 40.000, 40.400, 40.400, 42.250, 44.020, $44.730,44.900,46.300,50.330,51.442,57.220,58.700,58.800$, $61.200,61.740,65.440,65.597,66.000,74.100,75.800,84.100$, $106.600,109.700,121.970,121.970,185.560\}$.

The second dataset corresponds to the remission times (in months) of 128 bladder cancer patients chosen at random. Bladder cancer is a condition in which abnormal cells multiply uncontrollably in the bladder. Transitional cell carcinoma is the most common type of bladder cancer that mimics the normal histology of the urothelium. Zea and others [14], among others, had previously studied these data. The values of data are $\{0.08,0.20,0.40,0.50,0.51,0.81,0.90$, $1.05,1.19,1.26,1.35,1.40,1.46,1.76,2.02,2.02,2.07,2.09$, $2.23,2.26,2.46,2.54,2.62,2.64,2.69,2.69,2.75,2.83,2.87$, $3.02,3.25,3.31,3.36,3.36,3.48,3.52,3.57,3.64,3.70,3.82$, $3.88,4.18,4.23,4.26,4.33,4.34,4.40,4.50,4.51,4.87,4.98$, $5.06,5.09,5.17,5.32,5.32,5.34,5.41,5.41,5.49,5.62,5.71$, $5.85,6.25,6.54,6.76,6.93,6.94,6.97,7.09,7.26,7.28,7.32$,
$7.39,7.59,7.62,7.63,7.66,7.87,7.93,8.26,8.37,8.53,8.65$, $8.66,9.02,9.22,9.47,9.74,10.06,10.34,10.66,10.75,11.25$, $11.64,11.79,11.98,12.02,12.03,12.07,12.63,13.11,13.29$, $13.80,14.24,14.76,14.77,14.83,15.96,16.62,17.12,17.14$, $17.36,18.10,19.13,20.28,21.73,22.69,23.63,25.74,25.82$, $26.31,32.15,34.26,36.66,43.01,46.12,79.05\}$.

Table 4 and 5 provide values of maximum likelihood estimates and standard errors for all fitted distribution for two real data sets. In addition, these tables contain the values of $\log$-likelihood $(\operatorname{LogL})$, Akaike information criterion (AIC), Bayesian information criterion (BIC), and Kolmogorov-Smirnov (KS) statistic along with its $p$ value for all fitted distributions for two real data. The fitted PINH distribution, pdf, cdf, and PP-plot of the two real data are displayed in Figures 3 and 4, respectively.

The above Tables 4 and 5 show that the PINH distribution is the best fitted as compared with TINH, MOINH, and INH, as PINH distribution shows smaller values of 

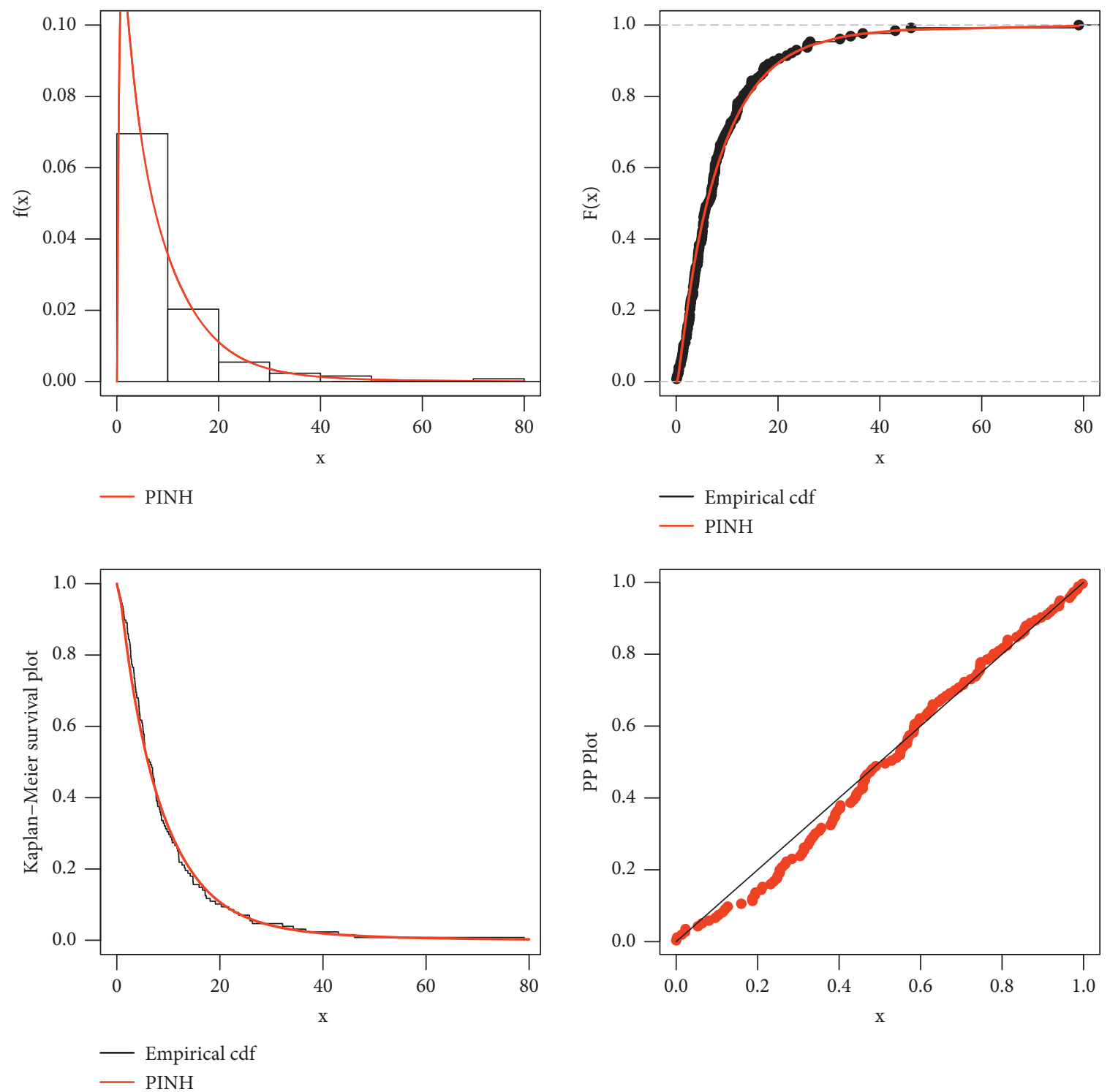

Figure 4: Fitted probability models and empirical data for second data set.

information criterions as compared with other distributions. The graph of estimated densities and cdf curves in Figures 3 and 4, respectively, shows that the introduced model of PINH distribution is the best fitted model.

\section{Conclusion}

In this work, we proposed a three-parameter distribution based on the INH distribution called PINH distribution and derived its mathematical properties. Many important mathematical properties are derived that allow us its application in many real-life situations. Further, entropies and order statistics are derived. The ML estimation method is used to obtain estimates of model parameters. From a simulation study, we observed that the ML estimator shows asymptotic efficiency and consistency. Additionally, we have shown the usefulness of the proposed distribution in a reallife application [15].

\section{Appendix}

\section{A}

Theorem 1. using Glanzel [12], suppose that the distribution function $G(x)$ defined over $(0 \infty)$ is characterized by the two truncated moments $q_{1}(x)$ and $q_{2}(x)$ such that

$$
E\left[q_{i}(x) \mid X>x\right]=\int_{x}^{\infty} q_{i}(t) \frac{f(t)}{1-G(x)} \mathrm{d} t, \quad i=1,2,
$$

and $E\left[q_{2}(x) \mid X \geq x\right] / E\left[q_{1}(x) \mid X \geq x\right]=\eta(x)$. Assume that the equation $\eta q_{1}=q_{2}$ has no real solution. That is, if the two functions $q_{1}(x)$ and $q_{2}(x)$ and $\eta(x)$ are known, then distribution $G(x)$ can be determined from

$$
G(x)=\int_{a}^{x} c\left|\frac{\eta^{\prime}(u)}{\eta(u) q_{1}(u)-q_{2}(u)}\right| e^{(-s(u))} \mathrm{d} u,
$$


where the function $s(u)$ is a solution of the differential equation.

$s^{\prime}(u)=\eta^{\prime}(u) q_{1}(u) / \eta(u) q_{1}(u)-q_{2}(u)$ and $c$ is the normalization constant.

\section{Data Availability}

Data are included in the manuscript.

\section{Conflicts of Interest}

The authors declare that they have no conflicts of interest.

\section{References}

[1] S. Nadarajah and F. Haghighi, "An extension of the exponential distribution," Statistics, vol. 45, no. 6, pp. 543-558, 2011.

[2] M. H. Tahir, G. M. Cordeiro, S. Ali, S. dey, and A. Manzoor, "The inverted Nadarajah-Haghighi distribution: estimation methods and applications," Journal of Statistical Computation and Simulation, vol. 88, no. 14, pp. 2775-2798, 2018.

[3] I. B. Abdul-Moniem, "Exponentiated nadarajah-haghighi exponential distribution," International Journal of Mathematical Analysis and Applications, vol. 2, no. 5, pp. 68-73, 2015.

[4] T. Vedo, A. D. C. Nascimento, W. R. Miranda Filho, M. C. S. Lima, L. G. B. Pinho, and G. M. Cordeiro, "Some computational and theoretical aspects of the Exponentiated Generalized Nadarajah-Haghighi distribution," Chilean Journal of Statistics, vol. 10, no. 1, pp. 1-25, 2016.

[5] H. M. Yousof and M. C. Korkmaz, "Topp-leone nadarajahhaghighi distribution," Journal of Statisticians: Statistics and Actuarial Sciences, vol. 2, no. 1, pp. 119-128, 2017.

[6] A. A. Ogunde, A. G. Ibraheem, and A. T. Audu, "Performance rating of transmuted Nadarajah and Haghighi exponential distribution: an analytical approach," Journal of Statistics: Advances in Theory and Applications, vol. 17, no. 2, pp. 137-151, 2017.

[7] C. R. Dias, M. Alizadeh, and G. M. Cordeiro, "The beta Nadarajah-Haghighi distribution," Hacettepe Journal of Mathematics and Statistics, vol. 47, no. 5, pp. 1302-1320, 2018.

[8] A. Toumaj, S. M. T. K. MirMostafaee, and G. G. Hamedani, "The transmuted inverted Nadarajah-Haghighi distribution with an application to lifetime data," Pakistan Journal of Statistics and Operation Research, vol. 17, no. 2, pp. 451-466, 2021.

[9] G. Raffiq, I. S. Dar, M. Ahsan-ul-Haq, and E. Ramos, "The Marshall-Olkin inverted Nadarajah-Haghighi distribution: estimation and applications," Annals of Data Science, pp. 1-16, 2020.

[10] P. Erdos and A. Renyi, "On random graphs," Publicationes Mathematicae, vol. 6, pp. 290-297, 1959.

[11] M. Kayid and S. Izadkhah, "Mean inactivity time function, associated orderings, and classes of life distributions," IEEE Transactions on Reliability, vol. 63, no. 2, pp. 593-602, 2014.

[12] W. A. Glanzel, "Characterization theorem based on truncated moments and its application to some distribution families," Theory of Probability and Mathematical Statistics, pp. 75-84, 1987.
[13] M. A. J. Van Montfort, "On testing that the distribution of extremes is of type I when type II is the alternative," Journal of Hydrology, vol. 11, no. 4, pp. 421-427, 1970.

[14] L. M. Zea, R. B. Silva, M. Bourguignon, A. M. Santos, and G. M. Cordeiro, "The beta exponentiated Pareto distribution with application to bladder cancer susceptibility," International Journal of Statistics and Probability, vol. 1, no. 2, p. 8, 2012.

[15] R. C. Gupta, P. L. Gupta, and R. D. Gupta, "Modeling failure time data by Lehman alternatives," Communications in Statistics - Theory and Methods, vol. 27, no. 4, pp. 887-904, 1998. 